\title{
Short communication: Comparative gene expression analysis on the enrichment of polymorphonuclear leukocytes and gastrointestinal epithelial cells in fecal RNA from nondiarrheic neonatal dairy calves
}

\section{F. Rosa and J. S. Osorio*}

Department of Dairy and Food Sciences, South Dakota State University, Brookings 57007

\section{ABSTRACT}

Increased understanding of the biology of the gastrointestinal tract (GIT) in neonatal dairy calves during their adaptation to an extrauterine environment will decrease health problems such as diarrhea while increasing feed efficiency and average daily gain in preweaned dairy calves. Within this context, a noninvasive method, based on fecal RNA, to study the GIT in neonatal dairy calves through the isolation of RNA from fecal samples for quantitative reverse-transcription PCR analysis can provide valuable information on GIT biological adaptations during the preweaning period. We aimed to evaluate the potential enrichment of RNA from immune cells or GIT epithelial cells during fecal RNA isolation. Eight neonatal Holstein calves less than 3 wk old $(14.9 \pm 5.5 \mathrm{~d}$ of age at sampling \pm standard deviation) and a fecal score of $2.0 \pm 0.7$ (mean \pm standard deviation) were used. During a single sampling, fecal and blood samples were taken simultaneously from each calf before the morning feeding. Fecal samples were immediately frozen in liquid nitrogen until RNA isolation, whereas polymorphonuclear leukocytes (PMN) were isolated from blood samples before RNA isolation. An quantitative reverse-transcription PCR analysis was performed using a single standard curve composited of equal amounts of all samples including cDNA from fecal and PMN. The genes myeloperoxidase $(M P O)$ and L-selectin $(S E L L)$ were selected for their specific known function in PMN, whereas keratin 8 (KRT8) and aquaporin 3 (AQP3) have been associated with epithelial enterocytes. Our results showed a contrasting gene expression profile between PMN and fecal RNA; whereas greater mRNA expression of $S E L L$ was observed in PMN, a greater KRT8 expression was observed in fecal RNA. The mRNA expression of AQP3 tended to be greater in PMN than fecal RNA. Ad-

Received November 30, 2018.

Accepted April 19, 2019.

*Corresponding author: Johan.Osorio@sdstate.edu ditionally, $M P O$ was not amplified in fecal RNA. Our findings suggest that under nondiarrheic conditions RNA isolated from stool samples of neonatal dairy calves will have a considerable number of GIT epithelial cells, which confirms the reliability of this method under these conditions. However, further research needs to be done to determine if the same effects are observed during diarrhea or throughout the preweaning period of dairy calves.

Key words: fecal RNA, gene expression, polymorphonuclear leukocyte, dairy calf

\section{Short Communication}

The gastrointestinal tract (GIT) is a complex system with multiple functions including digestion and absorption of nutrients, protecting the host from a constant presence of microorganisms, toxins, and other chemicals in the lumen. In newborn calves, such functions and complexity are well known to be partially influenced by exogenous nutritional and nonnutritional factors (i.e., bioactive compounds) that are ingested with colostrum (Ontsouka et al., 2004). The latter effect is primarily associated with a direct influence on the maturation of the GIT in early life (Ontsouka et al., 2016). Therefore, understanding the progression of the GIT to a mature state, and determining what bioactive compounds in the colostrum have a predominant influence on this effect, are high priorities to minimize diarrhea and improve welfare among young dairy calves. However, studying early life maturation of the GIT can be challenging if this is done primarily through analysis and characterization of postmortem or surgical collections of GIT specimens. Such a challenge is more evident when repeated samplings over time on the same animals are desired to observe changes over time as well as to minimize animal variation. To overcome such challenges, we have previously proposed a novel technique by isolating RNA from fecal samples of newborn dairy calves with the aim of obtaining biological information on the GIT over time and using the same animals (Rosa et al., 
2018). Results from that study suggested that isolating RNA from fecal samples for subsequent quantitative reverse-transcription PCR analysis is a promising technique, where we observed biological adaptations of the young ruminant GIT over time to a mild diarrhea event, and these data were confirmed through blood biomarkers for inflammation and oxidative stress (Rosa et al., 2018). Although this technique is promising, several optimizations and validation steps are required to improve accuracy and robustness. For instance, the migration of PMN across the intestinal epithelium is a common pathological event of many mucosal inflammatory diseases (Brazil et al., 2010). Because the fecal RNA method described by Rosa et al. (2018) discriminated primarily against prokaryotic RNA (i.e., microbial RNA) but not immune cells, it is important to understand the potential influence immune cells such as PMN might have during fecal RNA analysis. Additionally, the fecal RNA method is based on the premise that stool samples contain a considerable number of epithelial cells (Nishizawa et al., 2005). Consequently, evaluating the RNA enrichment from immune_cells during fecal RNA isolation is essential to understand the reliability of this method. Therefore, the objective of this study was to evaluate the potential enrichment of RNA from PMN or GIT epithelial cells during RNA isolation from fecal samples of neonatal dairy calves. The Institutional Animal Care and Use Committee of South Dakota State University approved all procedures for this study (protocol no. A3958-01). During a single sampling, fecal and blood samples were taken simultaneously from 8 neonatal Holstein calves less than 3 wk old $(14.9 \pm 5.5 \mathrm{~d}$ of age at sampling $\pm \mathrm{SD})$ with a mean fecal score [scale 1-4, 1: firm, well-formed (not hard); 4: liquid, splatters (Osorio et al., 2012)] of $2.0 \pm$ 0.7 (mean $\pm \mathrm{SD}$ ) at sampling. The fecal RNA isolation was conduct using the same protocol as explained in Rosa et al. (2018). Briefly, $\sim 200 \mathrm{mg}$ of fecal sample was used for RNA isolation using a Trizol (catalog no. 15596018, Ambion, Carlsbad, CA) based method along with the RNeasy Plus Mini Kit (Qiagen, catalog no. 74134), following the manufacturer's instructions with some modifications. The RNA was eluted in 50 $\mu \mathrm{L}$ of RNase-free water, and the quantity for all fecal samples was $378.8 \pm 192.3 \mathrm{ng} / \mu \mathrm{L}$ and purity $(260 / 280$ ratio) was $2.0 \pm 0.1$ determined via Nanodrop. In the case of PMN, the complete PMN isolation protocol is presented in the Supplemental File (https://doi.org/ 10.3168/jds.2018-16074), and this protocol has been previously described by Osorio et al. (2013). Briefly, $100 \mathrm{~mL}$ of blood was collected into evacuated tubes $(10 \mathrm{~mL}, \mathrm{BD}$ Vacutainer, Becton Dickinson, Franklin Lakes, NJ) containing solution A of trisodium citrate, citric acid, and dextrose. Samples were centrifuged at
$600 \times g$ for $30 \mathrm{~min}$ at $4^{\circ} \mathrm{C}$. The plasma, buffy coat, and approximately one-third of the red blood cells were discarded. The remaining sample was purified through a series of washing and centrifugation steps using deionized water, $5 \times \mathrm{PBS}$, and $1 \times \mathrm{PBS}$. An aliquot $(20$ $\mu \mathrm{L}$ ) obtained during the isolation process was used for PMN quantification and viability using a granulocyte primary antibody (CH138A, Veterinary Microbiology and Pathology, Washington State University, Pullman, WA) followed by a second antibody (Goat Anti-Mouse IgM, Human ads-PE, Southern Biotech, Birmingham, AL). All samples harvested and used for analysis contained more than $80 \%$ PMN and had at least $90 \%$ viability. After PMN isolation, RNA was extracted using the Trizol-based method along with the RNeasy Plus Mini Kit. The RNA was eluted in $50 \mu \mathrm{L}$ of RNase-free water, the quantity for all PMN samples was $55.6 \pm$ $32.7 \mathrm{ng} / \mu \mathrm{L}$, and the purity was $1.9 \pm 0.1$. The RNA integrity was assessed using the TapeStation (Agilent Technologies, Santa Clara, CA), and the final RNA integrity number was $5.0 \pm 2.6$.

Details regarding primer sequences information and sources are presented in Supplemental Table S1 (https: //doi.org/10.3168/jds.2018-16074). The cDNA synthesis and quantitative PCR were performed according to the protocol described by Rosa et al. (2018) with some modifications. Each cDNA was synthesized by reverse transcription using 500 and $100 \mathrm{ng}$ of RNA for fecal and PMN samples, respectively. The quantitative PCR reaction was performed in a QuantStudio 6 Flex RealTime PCR System (Applied Biosystems, Waltham, MA) in a MicroAmp Optical 384-well Reaction Plate (Applied Biosystems). Relative mRNA expression of each gene was calculated based on a 6-point relative standard curve plus the nontemplate control. Such a standard curve was composited from all samples including cDNA from fecal and PMN. The internal control genes (ICG) used in this experiment were golgin subfamily A, member 5 (GOLGA5), oxysterol-binding protein-like 2 (OSBPL2), single-strand-selective monofunctional uracil-DNA glycosylase 1 (SMUG1), $\beta-2-$ microglobulin $(B 2 M), \beta$-actin $(A C T B)$, glyceraldehyde 3-phosphate dehydrogenase $(G A P D H)$, and ribosomal protein 9 (RPS9). Additionally, the ribosomal protein S15A (RPS15A) and peptidylprolyl isomerase A (PPIA) were evaluated as potential ICG, but they were deemed to have low stability across samples. Therefore, the geometric mean of the 7 ICG was used to normalize the expression of the target genes on both types of samples (i.e., fecal and PMN). The stability of the ICG was assessed using the geNorm software (Vandesompele et al., 2002), with a favorable final pairwise variation of 0.19. The ribosomal protein S15A $(R P S 15 A)$ and peptidylprolyl isomerase A $(P P I A)$ were evaluated as 
Table 1. Expression of target genes associated with epithelial and immune cells in PMN and fecal samples from nondiarrheic neonatal dairy calves $(\mathrm{n}=8 /$ sample type $)$

\begin{tabular}{lcccc}
\hline & \multicolumn{3}{c}{ Sample type } & \\
Gene $^{1}$ & Fecal & PMN & SEM $^{2}$ & $P$-value \\
\hline KRT8 & 2.43 & -9.08 & 0.52 & $<0.01$ \\
AQP3 & -4.25 & -2.45 & 0.70 & 0.06 \\
MPO & - & -0.36 & 2.31 & - \\
SELL & -5.58 & -2.57 & 0.70 & 0.01 \\
\hline
\end{tabular}

${ }^{1}$ Target genes: $K R T 8=$ cytokeratine $8 ; A Q P 3=$ aquaporin $3 ; M P O=$ myeloperoxidase; $S E L L=$ selectin-L.

${ }^{2}$ Largest SEM.

${ }^{3} P$-values represent the probability of statistical significance in relative mRNA expression between fecal RNA and PMN samples.

${ }^{4}$ Standard deviation for MPO in PMN.

potential ICG, but they were deemed to be unsuitable due to low stability across samples. Normalized gene expression data were log-transformed before statistical analysis. The target genes were selected based on their known specific association with either PMN or GIT epithelial cells. In the case of PMN, the genes selected were myeloperoxidase $(M P O)$ and selectin-L $(S E L L)$, which are well-documented genes specific for PMN (Bionaz and Loor, 2008; Moyes et al., 2009; Batistel et al., 2017), whereas aquaporin 3 (AQP3; Ricanek et al., 2015) and keratin 8 (KRT8; Nishizawa et al., 2005) have been associated with epithelial enterocytes. Data were analyzed using the PROC MIXED procedure of SAS 9.4 (SAS Institute Inc., Cary, NC). The model included type (PMN or GIT) as fixed effect and calf as random. Statistical significance and tendencies were declared at $P \leq 0.05$ and $0.05 \leq P \leq 0.10$, respectively.

Keratins are the largest family of cellular intermediate filament proteins and are predominant in epithelial cells (Iyer et al., 2013), and among these, keratin 8 has been associated with mammary epithelial cells and small intestinal mucosa (Moll et al., 1982, 2008). In fact, the antibody against keratin 8 has been commonly used in ruminants for cell purification of mammary epithelial cells from total milk cells (Boutinaud et al., 2015). In contrast to epithelial cells, to the authors' knowledge, the expression of keratin 8 has not been reported in immune cells, and specifically PMN. Accordingly, our results showed a substantially lower $(P<0.01) \mathrm{mRNA}$ expression of KRT8 in PMN in comparison to fecal RNA (Table 1), which suggests that the fecal RNA samples contained a greater number of GIT epithelial cells. Interestingly, this is supported by KRT8 having the greatest mRNA abundance in fecal RNA (Table 2), in contrast to PMN, where SELL occupied the greatest mRNA abundance.

The genes $M P O$ and SELL encode the enzyme myeloperoxidase and the cell membrane L-selectin, respectively, and are essential for the normal cellular functions associated with PMN cells in bovine (Paape et al., 2003). Myeloperoxidase is required by PMN to produce reactive oxygen species responsible for the oxidative burst and consequently killing capacity of PMN, whereas L-selectin allows PMN to recognize sites

Table 2. Slope, coefficient of determination of the standard curve $\left(\mathrm{R}^{2}\right)$, efficiency (E) of amplification, and median cycle threshold $(\mathrm{Ct})$ of quantitative reverse-transcription PCR

\begin{tabular}{|c|c|c|c|c|c|}
\hline Gene & Slope $^{1}$ & $\mathrm{R}^{2}$ & $\mathrm{E}^{2}$ & $\mathrm{Ct}^{3}$ & $\begin{array}{c}\text { mRNA } \\
\text { abundance }^{4}(\%)\end{array}$ \\
\hline \multicolumn{6}{|c|}{ All samples } \\
\hline SELL & -3.459 & 0.98 & 1.95 & 21.90 & 99.38 \\
\hline AQP3 & -3.171 & 0.99 & 2.07 & 29.93 & 0.48 \\
\hline KRT8 & -2.902 & 0.99 & 2.21 & 32.41 & 0.13 \\
\hline$M P O$ & -2.076 & 0.95 & 3.03 & 32.19 & 0.00 \\
\hline \multicolumn{6}{|c|}{ PMN samples } \\
\hline SELL & -3.459 & 0.98 & 1.95 & 21.46 & 99.55 \\
\hline$A Q P 3$ & -3.171 & 0.99 & 2.07 & 29.05 & 0.44 \\
\hline KRT8 & -2.902 & 0.99 & 2.21 & 33.12 & 0.01 \\
\hline$M P O$ & -2.076 & 0.95 & 3.03 & 31.87 & 0.00 \\
\hline \multicolumn{6}{|c|}{ Fecal samples } \\
\hline SELL & -3.459 & 0.98 & 1.95 & 29.91 & 24.74 \\
\hline$A Q P 3$ & -3.171 & 0.99 & 2.07 & 35.60 & 0.10 \\
\hline KRT8 & -2.902 & 0.99 & 2.21 & 27.03 & 75.15 \\
\hline$M P O$ & -2.076 & 0.95 & 3.03 & - & 0.00 \\
\hline
\end{tabular}

${ }^{1}$ Slope of the 6-point standard curve.

${ }^{2}$ Efficiency of amplification $\left[\mathrm{E}=10^{(-1 / \text { slope })}\right]$.

${ }^{3} \mathrm{Ct}=$ median cycle threshold, which is defined as the number of cycles required for the fluorescent signal to cross the threshold (i.e., exceed background level), and the amount of target nucleic acid in the sample is inversely correlated to $\mathrm{Ct}$ cycles (i.e., the greater the amount of target nucleic acid, the lower the number of $\mathrm{Ct}$ cycles that will be required).

${ }^{4} \mathrm{mRNA}$ abundance is calculated as a percentage of $\left(1 / \mathrm{E}^{\Delta \mathrm{Ct}}\right)$ specific gene in the sum $\left(1 / \mathrm{E}^{\Delta \mathrm{Ct}}\right)$ all genes. 
of infection or inflammation (Paape et al., 2003). Our results showed a lower $(P=0.01)$ mRNA expression of $S E L L$ and a lack of amplification of $M P O$ in fecal RNA samples in comparison to the PMN samples (Table 1). Therefore, it is conceivable that the fecal RNA samples contained small amounts of PMN mRNA transcripts, which is in agreement with the greater expression of KRT8 in fecal RNA.

Among the target genes in this study, MPO had a greater efficiency than normal $(>3$; Table 2$)$, which could be associated with PCR amplification issues and indicates that the amplification, unrealistically, had more than a 1-fold increase during every cycle amplification. The authors associated this with the complete lack of amplification of $M P O$ in fecal samples, which might have skewed the slope and consequently the amplification efficiency.

Aquaporins are important cell transporters of water across biological membranes, and aquaglyceroporins, a subtype of aquaporins, are capable of transport water, glycerol, urea, and other small uncharged solutes across cell membranes (Rojek et al., 2008). Aquaporin 3 (encoded by $A Q P 3)$ is an aquaglyceroporin with a wide tissue distribution including kidney, skin, esophagus, spleen, and small intestine among others (Rojek et al., 2008). However, AQP3 is seldom reported to be expressed in PMN, and this aquaglyceroporin has been suggested to play a role in the uptake of microbes during phagocytosis (Marchini et al., 2003). In contrast to PMN, more data are available confirming the expression of $A Q P 3$ in enterocytes and, furthermore, describing a role for $A Q P 3$ during bacterial-induced diarrhea events in humans (Ricanek et al., 2015) and rats (Zhao et al., 2014). We recently demonstrated the amplification of $A Q P 3$ in fecal RNA in neonatal dairy calves, which also demonstrated a downregulation during a mild diarrhea event (Rosa et al., 2018). Interestingly, the current results demonstrate a trend $(P=0.06)$ for a greater expression of $A Q P 3$ in PMN in comparison to fecal RNA. Due to the lack of functional data on AQP3 in PMN, the latter effect remains unclear; however, the expression of other aquaglyceroporins such as AQP9 have been reported in peripheral leukocytes (Ishibashi et al., 1998).

Taking these data together, we observed a contrasting gene expression profile between PMN and fecal RNA; whereas the greater mRNA expression of $S E L L$ in PMN was expected, the greater mRNA expression of KRT8 in fecal RNA suggests that a considerable amount of RNA originated from epithelial cells in fecal RNA. These data provide new evidence on the reliability of the fecal RNA method; however, the data are not conclusive and further research needs to be done to improve the accuracy and robustness of this method while determining its limitations.

The data presented here are nuances in the refinement of the fecal RNA method to study biological adaptations of the GIT of neonatal dairy calves through analysis of transcriptional alterations. Our findings suggest that under nondiarrheic conditions RNA isolated from stool samples of neonatal dairy calves will have a considerable number of GIT epithelial cells, which confirms the reliability of this method under these conditions. However, further research needs to be done to determine if the same effects are observed during diarrhea or throughout the preweaning period of dairy calves.

\section{ACKNOWLEDGMENTS}

This work was supported by the South Dakota Agricultural Experimental Station under project no. SD00H612-16. We gratefully acknowledge the help from the staff of South Dakota State University Dairy Research and Teaching Facility for animal care and handling.

\section{REFERENCES}

Batistel, F., J. S. Osorio, M. R. Tariq, C. Li, J. Caputo, M. T. Socha, and J. J. Loor. 2017. Peripheral leukocyte and endometrium molecular biomarkers of inflammation and oxidative stress are altered in peripartal dairy cows supplemented with $\mathrm{Zn}, \mathrm{Mn}$, and $\mathrm{Cu}$ from amino acid complexes and Co from Co glucoheptonate. J. Anim. Sci. Biotechnol. 8:33.

Bionaz, M., and J. J. Loor. 2008. Gene networks driving bovine milk fat synthesis during the lactation cycle. BMC Genomics 9:366.

Boutinaud, M., L. Herve, and V. Lollivier. 2015. Mammary epithelial cells isolated from milk are a valuable, non-invasive source of mammary transcripts. Front. Genet. 6:323.

Brazil, J. C., W. Y. Lee, K. N. Kolegraff, A. Nusrat, C. A. Parkos, and N. A. Louis. 2010. Neutrophil migration across intestinal epithelium: Evidence for a role of CD44 in regulating detachment of migrating cells from the luminal surface. J. Immunol. 185:7026-7036.

Ishibashi, K., M. Kuwahara, Y. Gu, Y. Tanaka, F. Marumo, and S. Sasaki. 1998. Cloning and functional expression of a new aquaporin (AQP9) abundantly expressed in the peripheral leukocytes permeable to water and urea, but not to glycerol. Biochem. Biophys. Res. Commun. 244:268-274.

Iyer, S. V., P. P. Dange, H. Alam, S. S. Sawant, A. D. Ingle, A. M. Borges, N. V. Shirsat, S. N. Dalal, and M. M. Vaidya. 2013. Understanding the role of keratins 8 and 18 in neoplastic potential of breast cancer derived cell lines. PLoS One 8:e53532.

Marchini, G., B. Stabi, K. Kankes, S. Lonne-Rahm, M. Ostergaard, and S. Nielsen. 2003. AQP1 and AQP3, psoriasin, and nitric oxide synthases 1-3 are inflammatory mediators in erythema toxicum neonatorum. Pediatr. Dermatol. 20:377-384.

Moll, R., M. Divo, and L. Langbein. 2008. The human keratins: Biology and pathology. Histochem. Cell Biol. 129:705-733.

Moll, R., W. W. Franke, D. L. Schiller, B. Geiger, and R. Krepler. 1982. The catalog of human cytokeratins: Patterns of expression in normal epithelia, tumors and cultured cells. Cell 31:11-24.

Moyes, K. M., J. K. Drackley, D. E. Morin, M. Bionaz, S. L. Rodriguez-Zas, R. E. Everts, H. A. Lewin, and J. J. Loor. 2009. Gene network and pathway analysis of bovine mammary tissue chal- 
lenged with Streptococcus uberis reveals induction of cell proliferation and inhibition of PPARgamma signaling as potential mechanism for the negative relationships between immune response and lipid metabolism. BMC Genomics 10:542.

Nishizawa, M., I. Izawa, A. Inoko, Y. Hayashi, K. Nagata, T. Yokoyama, J. Usukura, and M. Inagaki. 2005. Identification of trichoplein, a novel keratin filament-binding protein. J. Cell Sci. 118:1081-1090.

Ontsouka, E. C., C. Albrecht, and R. M. Bruckmaier. 2016. Invited review: Growth-promoting effects of colostrum in calves based on interaction with intestinal cell surface receptors and receptor-like transporters. J. Dairy Sci. 99:4111-4123.

Ontsouka, E. C., H. M. Hammon, and J. W. Blum. 2004. Expression of insulin-like growth factors (IGF)-1 and -2, IGF-binding proteins-2 and -3 , and receptors for growth hormone, IGF type- 1 and -2 and insulin in the gastrointestinal tract of neonatal calves. Growth Factors 22:63-69.

Osorio, J. S., E. Trevisi, M. A. Ballou, G. Bertoni, J. K. Drackley, and J. J. Loor. 2013. Effect of the level of maternal energy intake prepartum on immunometabolic markers, polymorphonuclear leukocyte function, and neutrophil gene network expression in neonatal Holstein heifer calves. J. Dairy Sci. 96:3573-3587.

Osorio, J. S., R. L. Wallace, D. J. Tomlinson, T. J. Earleywine, M. T. Socha, and J. K. Drackley. 2012. Effects of source of trace minerals and plane of nutrition on growth and health of transported neonatal dairy calves. J. Dairy Sci. 95:5831-5844.

Paape, M. J., D. D. Bannerman, X. Zhao, and J. W. Lee. 2003. The bovine neutrophil: Structure and function in blood and milk. Vet. Res. 34:597-627.

Ricanek, P., L. K. Lunde, S. A. Frye, M. Stoen, S. Nygard, J. P. Morth, A. Rydning, M. H. Vatn, M. Amiry-Moghaddam, and T. Tonjum. 2015. Reduced expression of aquaporins in human intestinal mucosa in early stage inflammatory bowel disease. Clin. Exp. Gastroenterol. 8:49-67.

Rojek, A., J. Praetorius, J. Frokiaer, S. Nielsen, and R. A. Fenton. 2008. A current view of the mammalian aquaglyceroporins. Annu. Rev. Physiol. 70:301-327.

Rosa, F., S. Busato, F. C. Avaroma, K. Linville, E. Trevisi, J. S. Osorio, and M. Bionaz. 2018. Transcriptional changes detected in fecal RNA of neonatal dairy calves undergoing a mild diarrhea are associated with inflammatory biomarkers. PLoS One 13:e191599.

Vandesompele, J., K. De Preter, F. Pattyn, B. Poppe, N. Van Roy, A. De Paepe, and F. Speleman. 2002. Accurate normalization of real-time quantitative RT-PCR data by geometric averaging of multiple internal control genes. Genome Biol. 3:RESEARCH0034.

Zhao, G., J. Li, J. Wang, X. Shen, and J. Sun. 2014. Aquaporin 3 and 8 are down-regulated in TNBS-induced rat colitis. Biochem. Biophys. Res. Commun. 443:161-166. 